\title{
Monochromatic and mean radiative properties of astrophysical plasma mixtures in nonlocal thermodynamic equilibrium regime
}

\author{
Rafael Rodríguez ${ }^{1,2}$ (D) | Guadalupe Espinosa ${ }^{1} \mid$ Juan Miguel Gil $^{1,2} \mid$ Pablo R. Beltrán ${ }^{1}$
}

${ }^{1}$ Department of Physics, IUNAT, Universidad de Las Palmas de Gran Canaria, Las Palmas, Spain

${ }^{2}$ Instituto de Fusión Nuclear, Universidad Politécnica de Madrid, Madrid, Spain

\section{Correspondence}

Rafael Rodríguez, Department of Physics, IUNAT, Universidad de Las Palmas de Gran Canaria, Las Palmas, Spain. Email: rafael.rodriguezperez@ulpgc.es

\section{Funding information}

EUROfusion, Grant/Award Number: Enabling Research IFE, Project No. AWP15-ENR-01/CE; Spanish Government, Grant/Award Number: FIS201681019-P; Project of the Spanish Government, Grant/Award Number: FIS201681019-P; EUROfusion Consortium Task Agreement WPENR: Enabling Research IFE Project, Grant/Award Number: AWP15-ENR-01/CEA-02
Radiative-hydrodynamics and radiative transfer simulations of astrophysical plasmas require the determination of radiative properties. However, most of the plasma radiative properties are calculated assuming the plasma in coronal equilibrium or local thermodynamic equilibrium regimes that is often not the case for many scenarios. In this work, we present nonlocal thermodynamic equilibrium calculations of radiative opacities of $\mathrm{Fe}$ and $\mathrm{S}$ and of an astrophysical plasma mixture for temperatures larger than $100 \mathrm{eV}$. We also analyze the departure from local thermodynamic equilibrium simulations.

\section{1 | INTRODUCTION}

Radiative properties are essential in astrophysics. Thus, the opacities are fundamental in the radiative energy transfer affecting the structure of the star evolution ${ }^{[1]}$ and have a strong influence on radiative hydrodynamic simulations (RHS). On the other hand, the emissivity is a key feature because the emission spectra carry information about the density, temperature, and chemical composition of the radiating object. Furthermore, the behavior of the cooling rates with the temperature can lead to thermal instabilities ${ }^{[2,3]}$ of radiative shock waves that could be responsible for the origin of some astrophysical objects. Most of the opacity calculations are made in local thermodynamic equilibrium (LTE), which is accurate for high density conditions. On the other hand, the cooling coefficients are commonly obtained assuming coronal equilibrium, which is valid for very low density optically thin plasmas. However, in wide ranges of plasma conditions, the plasmas are in nonLTE (NLTE). The basis of NLTE simulations is the collisional-radiative models $(\mathrm{CRM})^{[4,5]}$ in which a large set of rate equations, including all relevant collisional and radiative atomic processes that populate and depopulate the plasma atomic levels, must be solved. Once the plasma level populations distributions are obtained, the radiative properties can be calculated. NLTE simulations are much more complicated than the LTE and coronal equilibrium because there is not a priori expression for the occupation probabilities of the atomic levels. Many works have been devoted to the calculation and analysis of both multicomponent and monocomponent plasmas of astrophysical interest but predominantly assuming LTE regime. ${ }^{[6-10]}$ However, there is a relative lack of 
studies of the radiative properties of these plasmas in the NLTE regime.

The objective of this work is twofold: first, to analyze the NLTE effects in the simulation of plasma monochromatic and mean opacities of astrophysical plasma mixtures and second, to study the departures from calculations assuming LTE regime. The elements considered in the mixture were $\mathrm{H}, \mathrm{He}, \mathrm{C}, \mathrm{N}, \mathrm{O}, \mathrm{Ne}, \mathrm{Na}, \mathrm{Mg}, \mathrm{Al}$, $\mathrm{Si}, \mathrm{S}, \mathrm{Ar}, \mathrm{Ca}$, and Fe. Solar-like abundances provided by Asplund et al. ${ }^{[11]}$ were used. Although the electron densities and temperatures can be simulated within a range of $10^{7}-10^{20} \mathrm{~cm}^{-3}$ and $1-4,000 \mathrm{eV}$, respectively, we have limited the simulations to plasma temperatures larger than $100 \mathrm{eV}$ and electron densities ranged from $10^{16}$ to $10^{19} \mathrm{~cm}^{-3}$, in which the high $\mathrm{Z}$ elements of the mixture $(\mathrm{S}-\mathrm{Fe})$ are highly ionized, and the plasma is in NLTE regime. As far as we know, there is a lack of studies in the range of plasma conditions addressed in this work. In Section 2, we present a brief explanation of the theoretical models and atomic kinetics codes used to calculate the radiative properties. Section 3 is devoted to present the NLTE simulations and the comparisons with the LTE ones. Finally, the main conclusions are presented in Section 4.

\section{2 | THEORETICAL AND COMPUTATIONAL MODELS}

The atomic data used in the simulations were obtained from FAC code ${ }^{[12]}$ in which a fully relativistic approach based on the Dirac equation is used. In particular, from FAC, we obtained relativistic energy levels, wave functions, oscillator strengths (including a correction for mixing between levels belonging to the same nonrelativistic configuration), and photoionization cross sections (generated in the distorted wave approach). The radiative properties in FAC are obtained in the single multipole approximation and in this work were calculated in the electric dipole approach. The atomic data were generated in the relativistic detailed configuration approach for isolated ions. The effect of the plasma surrounding on the atomic properties and on the population of the atomic levels is modeled through the continuum lowering, using the model developed by Stewart and Pyatt. ${ }^{[13]}$ The set of atomic configurations included for each ion were those with energies within two times the ionization energy of its ground configuration.

For the calculation of the plasma level populations, MIXKIP code ${ }^{[14]}$ was used. The plasma was assumed optically thin in steady-state. The latter assumption is valid when the characteristic time of the most relevant atomic process in the plasma is considerably shorter than the characteristic time associated to changes of the density and temperature of the plasma. Therefore, for situations in which the plasma conditions evolve quickly over time, this approach may not be accurate enough. In LTE assumption, the plasma level populations are obtained from the Saha-Boltzmann equations. For NLTE calculations, in MIXKIP, the rate equations of a CRM that includes the most relevant atomic processes (collisional ionization and three-body recombination, electro excitation and deexcitation, spontaneous decay, autoionization, electron capture, and radiative recombination) are solved. In MIXKIP, it is assumed that the ions are at rest, which is valid when the collision times between ions and electrons are short enough. Furthermore, a Maxwell-Boltzmann distribution for the free electrons is assumed for the calculation of the rates of the atomic processes. The characteristic time for the electrons to thermalize is obtained from the electron self-collision time. ${ }^{[15]}$ For the range of plasma conditions studied in this work, we have calculated this time obtaining that is ranged from 2 ps to $5 \mathrm{~ns}$. Therefore, for those situations in which this characteristic time is larger than the characteristic time associated to changes of the density and temperature of the plasma, free electrons do not thermalize and their energy distribution functions are usually obtained from a simultaneous solution of the time-dependent atomic rate equations and Boltzmann electron kinetics. A more detailed explanation of the CRM implemented in MIXKIP can be found in Espinosa et al. ${ }^{[14]}$

Finally, monochromatic and mean radiative properties were calculated using RAPCAL code. ${ }^{[16,17]}$ Boundbound, bound-free, and free contributions were considered. In the evaluation of the line profiles in the bound-bound transitions, natural, Doppler, electron impact $^{[18]}$ and the unresolved transition array ${ }^{[19]}$ broadenings are included. As said before, the photoionization cross sections were obtained from FAC code, and for the free-free contribution, the Kramers semiclassical expression for the inverse bremsstrahlung cross section $^{[20]}$ was used. A detailed explanation of the calculation of the radiative properties can be found in other works. ${ }^{[16,17]}$

\section{3 | RESULTS AND DISCUSSION}

In the range of high temperatures, that is, larger than $100 \mathrm{eV}$, highly charged ions are found in the plasma mixture. The chemical elements with larger contribution to the emissivity and the opacity, in this range of plasma temperatures, are $\mathrm{S}, \mathrm{Ar}, \mathrm{Ca}$, and $\mathrm{Fe} .{ }^{[21]}$ In particular, 

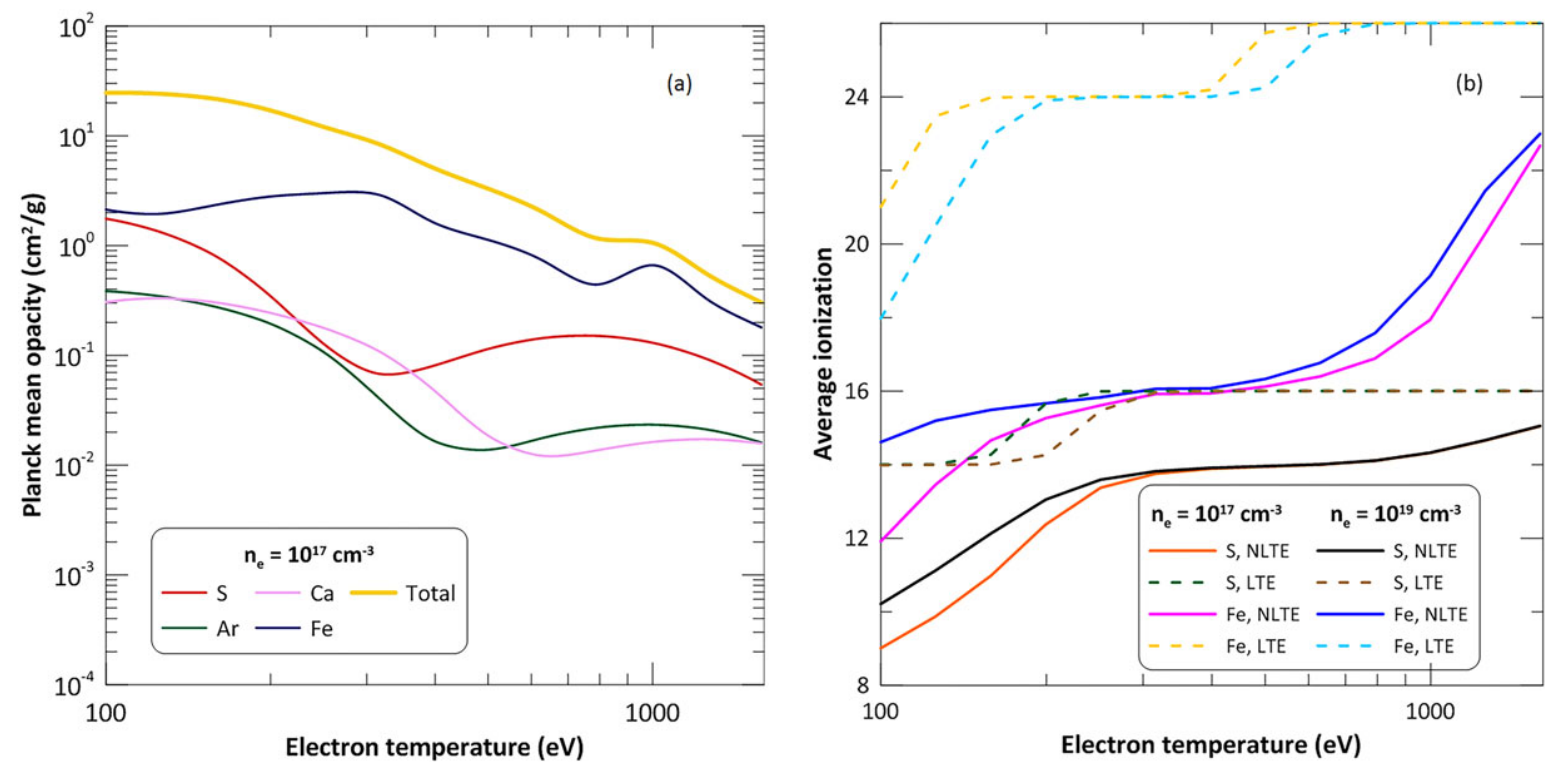

FIGURE 1 (a) Relative contribution of S, Ar, Ca, and Fe to the Planck mean opacity of the mixture. (b) LTE and NLTE calculations of the average ionization of S and Fe. NLTE: nonlocal thermodynamic equilibrium

Figure 1a shows that the elements that contribute most are $\mathrm{Fe}$ and $\mathrm{S}$, and their average ionizations ranged from 12 to 23 and from 9 to 15 for Fe and S, respectively (see Figure $1 \mathrm{~b}$ ). This figure also shows the great differences obtained between LTE and NLTE simulations of the average ionization. LTE model overestimates the average ionization, and this fact leads to great discrepancies in the radiative properties as well.

The differences in the radiative properties are well illustrated in Figure 2a,b where we have represented the Planck mean opacity and the radiative power loss, respectively, for S and Fe, calculated in LTE and NLTE. The figure shows significant differences for both properties that can reach several order of magnitudes for the mean opacity overall as the temperature increases and density decreases because both factors enhance the NLTE regime. Due to the larger average ionization predicted by the LTE simulations, the Planck mean opacities provided are lower than those obtained in NLTE simulations. In LTE simulations for the range of temperatures considered, both the mean opacity as the radiative power loss are ruled by $\mathrm{H}-$ and He-like ions, as it is portrayed in
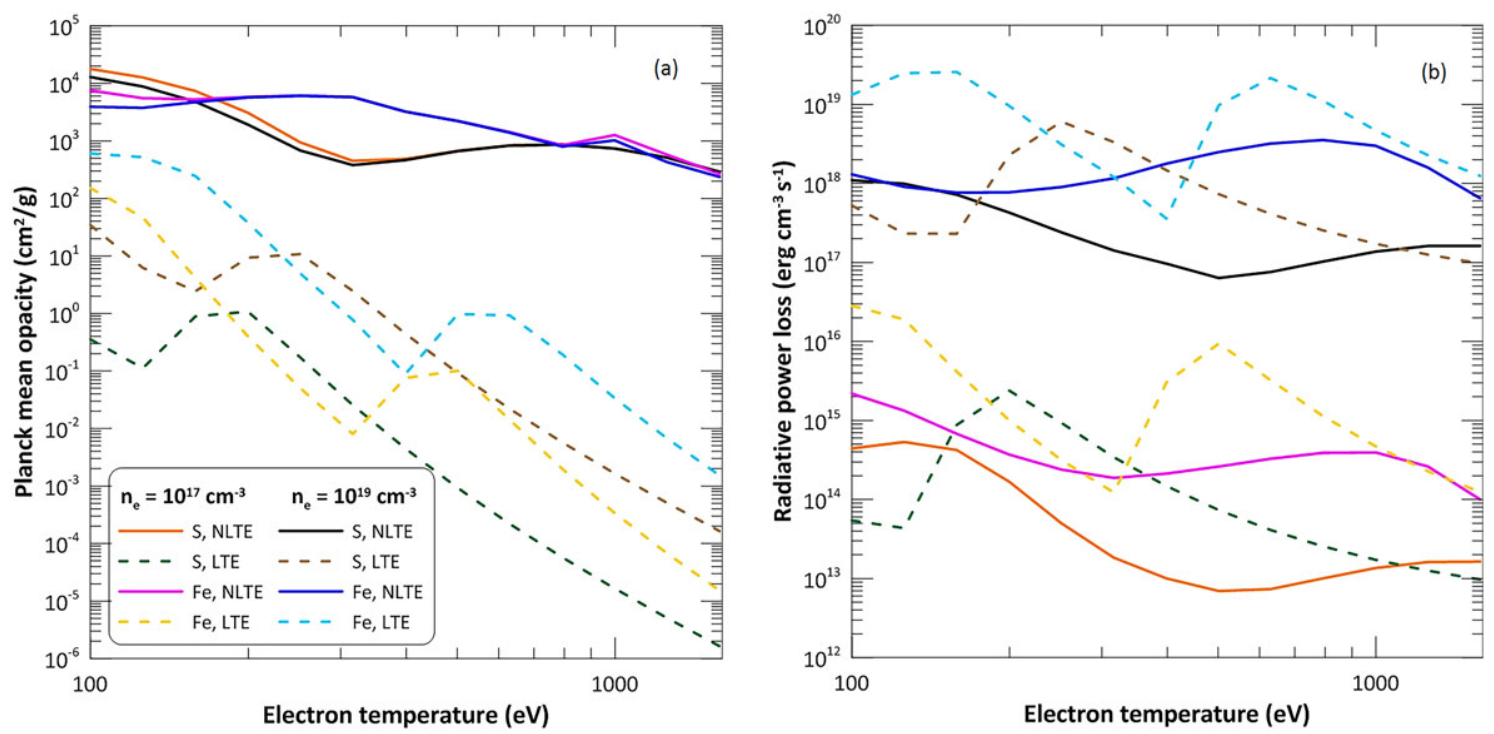

FIGURE 2 Comparison of LTE and NLTE simulations of (a) Planck mean opacity and (b) radiative power loss for S and Fe. NLTE: nonlocal thermodynamic equilibrium 


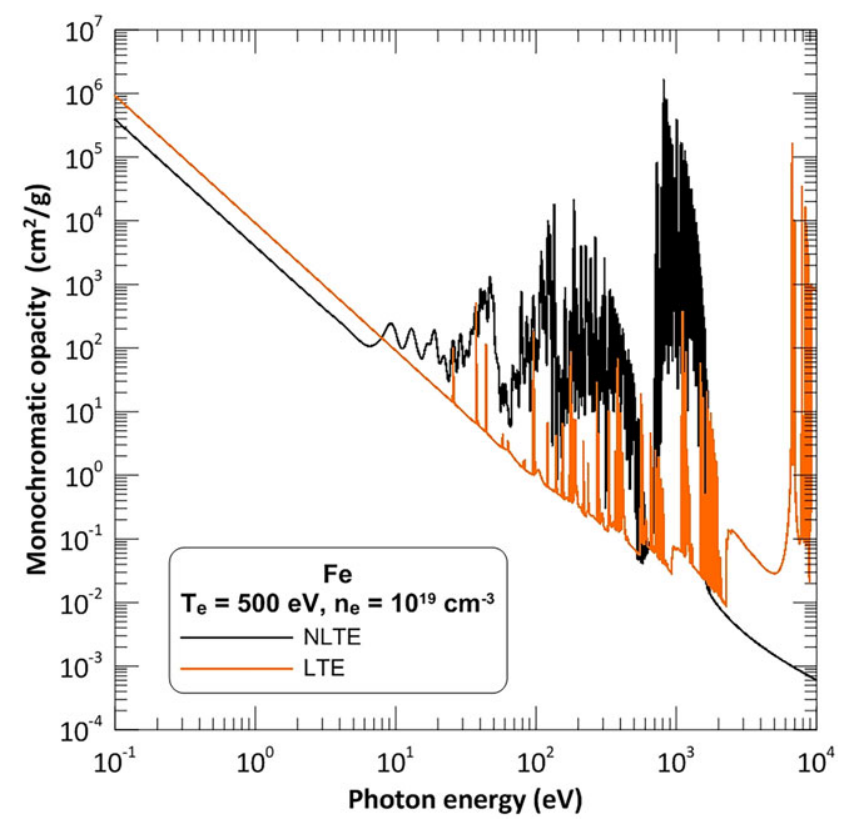

FIGURE 3 Comparison of LTE and NLTE simulations of monochromatic opacity for a plasma of Fe. NLTE: nonlocal thermodynamic equilibrium

Figure 2a,b. LTE simulations are more sensitive to the density than NLTE ones.

The analysis of the monochromatic opacities helps to understand such large discrepancies between LTE and NLTE mean opacities. In Figure 3, we have represented a comparison between the LTE and NLTE monochromatic opacities for an Fe plasma for electron density and temperature of $10^{19} \mathrm{~cm}^{-3}$ and $500 \mathrm{eV}$, respectively.
We detect that the line absorption structures in the LTE simulations are shifted toward higher photon energies than in the NLTE case due to the larger average ionization obtained in the former model, and therefore, significant differences are observed between both spectra. The figure shows that the low photon energies range (lower than $10 \mathrm{eV}$ ) is mainly due to the free-free contribution (i.e., the inverse bremsstrahlung). We also observe a large absorption in the X-ray spectra.

As said above, $\mathrm{S}$ and mainly Fe are the most relevant contributor to the astrophysical plasma mixture in the range of electron temperatures larger than $100 \mathrm{eV}$; therefore, the differences obtained between LTE and NLTE simulations for these elements will lead to great discrepancies in the plasma mixture. As an example, in Figure $4 \mathrm{a}$, we have represented a comparison of the Planck and Rosseland mean opacities calculated in both approaches. We detect that LTE Rosseland mean opacity is constant in almost the whole range of electron temperatures. This is due to Fe and S are fully stripped in that approach, and then Thomson scattering is the dominant contribution. The figure shows significant differences in the Plank mean opacity. LTE spectra are shifted toward high photon energies and present a small amount of line transitions due to the larger average ionization $(\mathrm{H}-$ and He-like ions are the most abundant ions in this approach), and Rosseland and Planck weighting functions peak around 1,500-2,000 eV of photon energies where LTE and NLTE spectra are quite different, as Figure $4 \mathrm{~b}$ shows. These facts explain the discrepancies between the mean opacities obtained with both models.
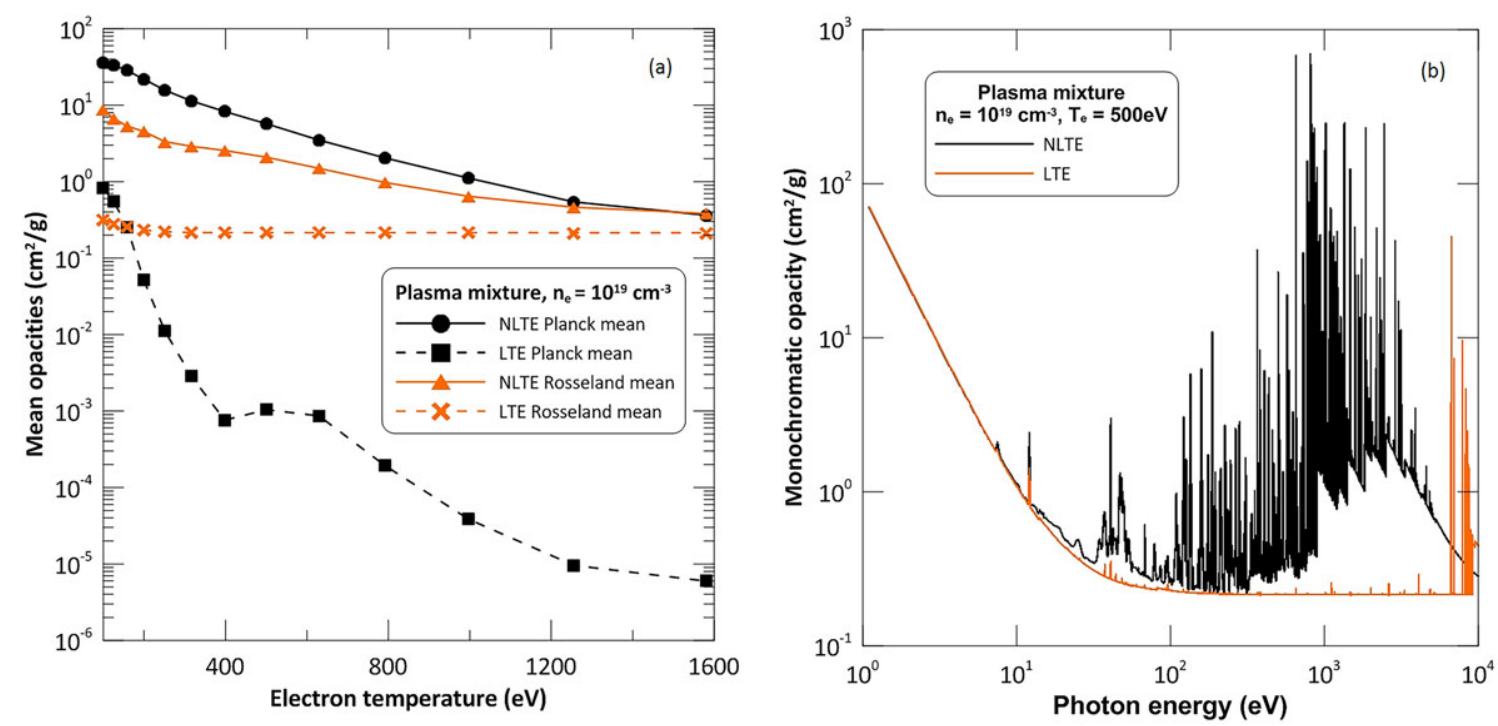

FIGURE 4 Comparisons between LTE and NLTE simulations of (a) mean and (b) monochromatic opacities, of the astrophysical plasma mixture. NLTE: nonlocal thermodynamic equilibrium 


\section{4 | CONCLUSIONS}

NLTE calculations of mean and monochromatic opacities of plasmas of $\mathrm{S}$ and Fe have been performed for electron temperatures larger than $100 \mathrm{eV}$ and lower than $1700 \mathrm{eV}$ because in this range of temperatures they are the most relevant contributors to a plasma astrophysical mixture. We have analyzed the departures from LTE simulations because this approach is commonly used in the RHS of astrophysical plasmas. We have found great differences that can even reach several order of magnitudes between the simulations obtained with both models that are mainly due to LTE calculations overestimate the plasma average ionization. As a result, significant discrepancies are obtained in the radiative properties for the plasma mixture, and this fact has to be taken into account in the RHS because inaccurate results or conclusions can be obtained. Finally, we have obtained that in this range of electron temperatures, the free-free contribution to the spectra is relevant in the range of low photon energies whereas the line transitions have a major contribution in the XUV and X-ray photon energy ranges.

\section{ACKNOWLEDGEMENTS}

This work has been supported by the EUROfusion Consortium Task Agreement WPENR: Enabling Research IFE Project AWP15-ENR-01/CEA-02 and by the Project of the Spanish Government with Reference FIS201681019-P.

\section{ORCID}

Rafael Rodríguez, (D) https://orcid.org/0000-0002-8326-3169

\section{REFERENCES}

[1] F. L. Wang, Z. Gang, J. M. Yuan, Astrophys. J. 2004, 600, 963.

[2] G. Field, Astrophys. J. 1965, 142, 531.

[3] J. J. Hunter, Astrophys. J. 1970, 161, 451.
[4] D. R. Bates, A. E. Kingston, R. P. W. McWhirter, Proc. R. Soc. London Ser. A 1962, 267, 297.

[5] R. W. P. McWhirter, Phys. Rep. 1978, 37, 165.

[6] N. Badnell, M. Bautista, K. Butler, Mon. Not. R. Astron. Soc. 2005, 360, 458.

[7] C. Igleisas, F. Rogers, Astrophys. J. Sup. Ser. 1996, 79, 507.

[8] J. Colgan, D. Kilcrease, N. Magee, M. Sherrill, J. Abdallah Jr., P. Hakel, C. Fontes, Astrophys. J. 2016, 817, 116.

[9] F. Jin, J. Zeng, T. Huang, Y. Ding, Z. Zheng, J. Yuan, Astrophys. J. 2009, 693, 597.

[10] S. Turck-Chieze, F. Delahaye, D. Gilles, G. Loisel, L. Piau, High Energy Density Phys. 2013, 9, 473.

[11] M. Asplund, N. Grevese, A. J. Sauval, P. Scott, Annu. Rev. Astron. Astrophys. 2009, 47, 481.

[12] M. F. Gu, Can. J. Phys. 2008, 86, 675.

[13] J. C. Stewart, K. D. Pyatt, Astrophys. J. 1966, 144, 1203.

[14] G. Espinosa, R. Rodríguez, J. M. Gil, F. Suzuki-Vidal, S. V. Lebedev, J. G. Rubiano, P. Martel, Phys. Rev. E 2017, 95. 033201

[15] L. Spitzer, Physics of fully ionized gases, Interscience, New York 1962.

[16] R. Rodríguez, R. Florido, J. M. Gil, J. G. Rubiano, P. Martel, E. Mínguez, Laser Part. Beams 2008, 26, 433.

[17] R. Rodríguez, R. Florido, J. M. Gil, J. G. Rubiano, D. Suárez, P. Martel, E. Mínguez, R. C. Mancini, Commun. Comput. Phys. 2010, 8, 185.

[18] M. S. Dimitrijevic, N. Konjevic, Astron. Astrophys. 1987, 172, 345.

[19] J. Bauche, C. Bauche-Arnoult, M. Klapisch, Adv. At. Mol. Phys. 1987, 23, 131.

[20] S. J. Rose, J. Phys. B 1992, 25, 1667.

[21] R. Rodríguez, G. Espinosa, J. M. Gil, Phys. Rev. E 2018, 98. 033213

How to cite this article: Rodríguez R, Espinosa G, Gil JM, Beltrán PR. Monochromatic and mean radiative properties of astrophysical plasma mixtures in nonlocal thermodynamic equilibrium regime. X-Ray Spectrometry. 2020;49:6-10. https:// doi.org/10.1002/xrs.2997 